\title{
Cluster expansion formulas and perfect matchings
}

\author{
Gregg Musiker • Ralf Schiffler
}

Received: 2 March 2009 / Accepted: 3 November 2009 / Published online: 19 November 2009

(C) Springer Science+Business Media, LLC 2009

\begin{abstract}
We study cluster algebras with principal coefficient systems that are associated to unpunctured surfaces. We give a direct formula for the Laurent polynomial expansion of cluster variables in these cluster algebras in terms of perfect matchings of a certain graph $G_{T, \gamma}$ that is constructed from the surface by recursive glueing of elementary pieces that we call tiles. We also give a second formula for these Laurent polynomial expansions in terms of subgraphs of the graph $G_{T, \gamma}$.
\end{abstract}

Keywords Cluster algebra · Triangulated surface · Principal coefficients · F-polynomial $\cdot$ Snake graph

\section{Introduction}

Cluster algebras, introduced in [17], are commutative algebras equipped with a distinguished set of generators, the cluster variables. The cluster variables are grouped into sets of constant cardinality $n$, the clusters, and the integer $n$ is called the rank of the cluster algebra. Starting with an initial cluster $\mathbf{x}=\left\{x_{1}, \ldots, x_{n}\right\}$ (together with a skew symmetrizable integer $n \times n$ matrix $B=\left(b_{i j}\right)$ and a coefficient vector $\mathbf{y}=\left(y_{i}\right)$

The first author is supported by an NSF Mathematics Postdoctoral Fellowship, and the second author is supported by the NSF grant DMS-0908765 and by the University of Connecticut.

G. Musiker ( $\triangleleft)$

Department of Mathematics, Room 2-336, Massachusetts Institute of Technology, 77 Massachusetts Ave., Cambridge, MA 02139, USA

e-mail: musiker@math.mit.edu

R. Schiffler

Department of Mathematics, University of Connecticut, 196 Auditorium Road, Storrs,

CT 06269-3009, USA

e-mail: schiffler@math.uconn.edu 
whose entries are elements of a torsion-free abelian group $\mathbb{P}$ ), the set of cluster variables is obtained by repeated application of so-called mutations. Note that this set may be infinite.

It follows from the construction that every cluster variable is a rational function in the initial cluster variables $x_{1}, x_{2}, \ldots, x_{n}$. In [17], it is shown that every cluster variable $u$ is actually a Laurent polynomial in the $x_{i}$, that is, $u$ can be written as a reduced fraction

$$
u=\frac{f\left(x_{1}, x_{2}, \ldots, x_{n}\right)}{\prod_{i=1}^{n} x_{i}^{d_{i}}},
$$

where $f \in \mathbb{Z} \mathbb{P}\left[x_{1}, x_{2}, \ldots, x_{n}\right]$ and $d_{i} \geq 0$. The right-hand side of (1) is called the cluster expansion of $u$ in $\mathbf{x}$.

The cluster algebra is determined by the initial matrix $B$ and the choice of the coefficient system. A canonical choice of coefficients is the principal coefficient system, introduced in [18], which means that the coefficient group $\mathbb{P}$ is the free abelian group on $n$ generators $y_{1}, y_{2}, \ldots, y_{n}$, and the initial coefficient vector $\mathbf{y}=\left\{y_{1}, y_{2}, \ldots, y_{n}\right\}$ consists of these $n$ generators. In [18], the authors show that knowing the expansion formulas in the case where the cluster algebra has principal coefficients allows one to compute the expansion formulas for arbitrary coefficient systems.

Inspired by the work of Fock and Goncharov [13-15] and Gekhtman, Shapiro, and Vainshtein $[22,23]$ which discovered cluster structures in the context of Teichmüller theory, Fomin, Shapiro, and Thurston [16, 20] initiated a systematic study of the cluster algebras arising from triangulations of a surface with boundary and marked points. In this approach, cluster variables in the cluster algebra correspond to arcs in the surface, and clusters correspond to triangulations. In [32], building on earlier results in $[31,33]$, this model was used to give a direct expansion formula for cluster variables in cluster algebras associated to unpunctured surfaces, with arbitrary coefficients, in terms of certain paths on the triangulation.

Our first main result in this paper is a new parameterization of this formula in terms of perfect matchings of a certain weighted graph that is constructed from the surface by recursive glueing of elementary pieces that we call tiles. To be more precise, let $x_{\gamma}$ be a cluster variable corresponding to an arc $\gamma$ in the unpunctured surface, and let $d$ be the number of crossings between $\gamma$ and the triangulation $T$ of the surface. Then $\gamma$ runs through $d+1$ triangles of $T$ and each pair of consecutive triangles forms a quadrilateral which we call a tile. So we obtain $d$ tiles, each of which is a weighted graph, whose weights are given by the cluster variables $x_{\tau}$ associated to the arcs $\tau$ of the triangulation $T$.

We obtain a weighted graph $G_{T, \gamma}$ by glueing the $d$ tiles in a specific way and then deleting the diagonal in each tile. To any perfect matching $P$ of this graph we associate its weight $w(P)$ which is the product of the weights of its edges, hence a product of cluster variables. We prove the following cluster expansion formula:

\section{Theorem 3.1}

$$
x_{\gamma}=\sum_{P} \frac{w(P) y(P)}{x_{i_{1}} x_{i_{2}} \ldots x_{i_{d}}},
$$


where the sum is over all perfect matchings $P$ of $G_{T, \gamma}, w(P)$ is the weight of $P$, and $y(P)$ is a monomial in $\mathbf{y}$.

We also give a formula for the coefficients $y(P)$ in terms of perfect matchings as follows. The $F$-polynomial $F_{\gamma}$, introduced in [18], is obtained from the Laurent polynomial $x_{\gamma}$ (with principal coefficients) by substituting 1 for each of the cluster variables $x_{1}, x_{2}, \ldots, x_{n}$. By [32, Theorem 6.2, Corollary 6.4], the $F$-polynomial has constant term 1 and a unique term of maximal degree that is divisible by all the other occurring monomials. The two corresponding matchings are the unique two matchings that have all their edges on the boundary of the graph $G_{T, \gamma}$. With respect to the construction of Sect. 3.2, $P_{-}$is the matching of $G_{T, \gamma}$ using the western edge of tile $\tilde{S}_{1}$. Now, for an arbitrary perfect matching $P$, the coefficient $y(P)$ is determined by the set of edges of the symmetric difference $P_{-} \ominus P=\left(P_{-} \cup P\right) \backslash\left(P_{-} \cap P\right)$ as follows.

Theorem 5.1 The set $P_{-} \ominus P$ is the set of boundary edges of a (possibly disconnected) subgraph $G_{P}$ of $G_{T, \gamma}$ which is a union of tiles $G_{P}=\bigcup_{j \in J} S_{j}$. Moreover,

$$
y(P)=\prod_{j \in J} y_{i_{j}} .
$$

Note that $y\left(P_{-}\right)=1$. As an immediate corollary, we see that the corresponding $g$-vector, introduced in [18], is

$$
g_{\gamma}=\operatorname{deg}\left(\frac{w\left(P_{-}\right)}{x_{i_{1}} \cdots x_{i_{d}}}\right) .
$$

Our third main result is yet another description of the formula of Theorem 3.1 in terms of the graph $G_{T, \gamma}$ only, see Theorem 6.1.

Theorem 3.1 has interesting intersections with work of other people. In [10], the authors obtained a formula for the denominators of the cluster expansion in types $A$, $D$, and $E$, see also [4]. In [5-7], an expansion formula was given in the case where the cluster algebra is acyclic and the cluster lies in an acyclic seed. Palu generalized this formula to arbitrary clusters in an acyclic cluster algebra [28]. These formulas use the cluster category introduced in [3], and in [9] for type $A$, and do not give information about the coefficients.

Recently, Fu and Keller generalized this formula further to cluster algebras with principal coefficients that admit a categorification by a 2-Calabi-Yau category [21], and, combining results of [1] and [2, 24], such a categorification exists in the case of cluster algebras associated to unpunctured surfaces.

In $[8,26,34,35]$, cluster expansions for cluster algebras of rank 2 are given; in [11, $19,30]$, the case $A$ is considered. In Sect. 4 of [30], Propp describes two constructions of snake graphs, the latter of which are unweighted analogues for the case A of the graphs $G_{T, \gamma}$ that we present in this paper. Propp assigns a snake graph to each arc in the triangulation of an $n$-gon and shows that the numbers of matchings in these graphs satisfy the Conway-Coxeter frieze pattern induced by the Ptolemy relations 
on the $n$-gon. In [25], a cluster expansion for cluster algebras of classical type is given for clusters that lie in a bipartite seed.

The formula for $y(P)$ given in Theorem 5.1 also can be formulated in terms of height functions, as found in literature such as [12] or [29]. We discuss this connection in Remark 5.3 of Sect. 5.

The paper is organized as follows. In Sect. 2, we recall the construction of cluster algebras from surfaces of [20]. Section 3 contains the construction of the graph $G_{T, \gamma}$ and the statement of the cluster expansion formula. Section 4 is devoted to the proof of the expansion formula. The formula for $y(P)$ and the formula for the $g$-vectors is given in Sect. 5. In Sect. 6, we present the expansion formula in terms of subgraphs and deduce a formula for the $F$-polynomials. We give an example in Sect. 7 .

\section{Cluster algebras from surfaces}

In this section, we recall the construction of [20] in the case of surfaces without punctures.

Let $S$ be a connected oriented two-dimensional Riemann surface with boundary and $M$ a nonempty finite set of marked points in the closure of $S$ with at least one marked point on each boundary component. The pair $(S, M)$ is called bordered surface with marked points. Marked points in the interior of $S$ are called punctures.

In this paper, we will only consider surfaces $(S, M)$ such that all marked points lie on the boundary of $S$, and we will refer to $(S, M)$ simply as an unpunctured surface.

We say that two curves in $S$ do not cross if they do not intersect each other except that endpoints may coincide.

Definition 1 An $\operatorname{arc} \gamma$ in $(S, M)$ is a curve in $S$ such that

(a) the endpoints are in $M$,

(b) $\gamma$ does not cross itself,

(c) the relative interior of $\gamma$ is disjoint from the boundary of $S$,

(d) $\gamma$ does not cut out a monogon or a digon.

Curves that connect two marked points and lie entirely on the boundary of $S$ without passing through a third marked point are called boundary arcs. Hence an arc is a curve between two marked points, which does not intersect itself nor the boundary except possibly at its endpoints and which is not homotopic to a point or a boundary arc.

Each arc is considered up to isotopy inside the class of such curves. Moreover, each arc is considered up to orientation, so if an arc has endpoints $a, b \in M$, then it can be represented by a curve that runs from $a$ to $b$, as well as by a curve that runs from $b$ to $a$.

For any two arcs $\gamma, \gamma^{\prime}$ in $S$, let $e\left(\gamma, \gamma^{\prime}\right)$ be the minimal number of crossings of $\gamma$ and $\gamma^{\prime}$, that is, $e\left(\gamma, \gamma^{\prime}\right)$ is the minimum of the numbers of crossings of arcs $\alpha$ and $\alpha^{\prime}$, where $\alpha$ is isotopic to $\gamma$, and $\alpha^{\prime}$ is isotopic to $\gamma^{\prime}$. Two arcs $\gamma, \gamma^{\prime}$ are called compatible if $e\left(\gamma, \gamma^{\prime}\right)=0$. A triangulation of $S$ is a maximal collection of compatible arcs together with all boundary arcs. The arcs of a triangulation cut the surface into 
Table 1 Examples of unpunctured surfaces

\begin{tabular}{llll}
\hline$b$ & $g$ & $m$ & surface \\
\hline 1 & 0 & $n+3$ & polygon \\
1 & 1 & $n-3$ & torus with disk removed \\
1 & 2 & $n-9$ & genus 2 surface with disk removed \\
2 & 0 & $n$ & annulus \\
2 & 1 & $n-6$ & torus with 2 disks removed \\
2 & 2 & $n-12$ & genus 2 surface with 2 disks removed \\
3 & 0 & $n-3$ & pair of pants \\
\hline
\end{tabular}

triangles. Since $(S, M)$ is an unpunctured surface, the three sides of each triangle are distinct (in contrast to the case of surfaces with punctures). Any triangulation has $n+m$ elements, $n$ of which are arcs in $S$, and the remaining $m$ elements are boundary arcs. Note that the number of boundary arcs is equal to the number of marked points. Each arc will correspond to a cluster variable, whereas each boundary arc will correspond to the multiplicative identity 1 in the cluster algebra.

Proposition 2.1 The number $n$ of arcs in any triangulation is given by the formula $n=6 g+3 b+m-6$, where $g$ is the genus of $S, b$ is the number of boundary components, and $m=|M|$ is the number of marked points. The number $n$ is called the rank of $(S, M)$.

Proof [20, 2.10].

Note that $b>0$ since the set $M$ is not empty. Table 1 gives some examples of unpunctured surfaces.

Following [20], we associate a cluster algebra to the unpunctured surface $(S, M)$ as follows. Choose any triangulation $T$, let $\tau_{1}, \tau_{2}, \ldots, \tau_{n}$ be the $n$ interior $\operatorname{arcs}$ of $T$, and denote the $m$ boundary arcs of the surface by $\tau_{n+1}, \tau_{n+2}, \ldots, \tau_{n+m}$. For any triangle $\Delta$ in $T$, define the matrix $B^{\Delta}=\left(b_{i j}^{\Delta}\right)_{1 \leq i \leq n, 1 \leq j \leq n}$ by

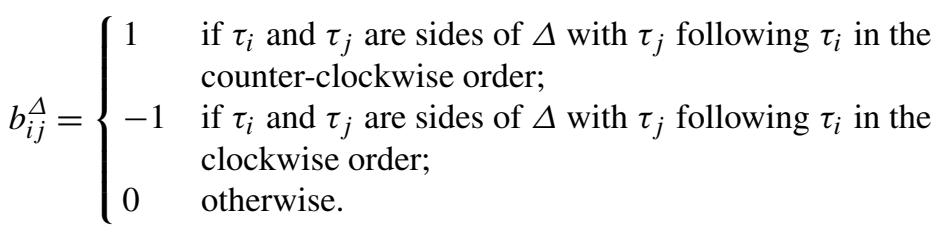

Note that this matrix is the transpose of the matrix defined in [20]. Then define the matrix $B_{T}=\left(b_{i j}\right)_{1 \leq i \leq n, 1 \leq j \leq n}$ by $b_{i j}=\sum_{\Delta} b_{i j}^{\Delta}$, where the sum is taken over all triangles in $T$. Note that the boundary arcs of the triangulation are ignored in the definition of $B_{T}$. Let $\tilde{B}_{T}=\left(b_{i j}\right)_{1 \leq i \leq 2 n, 1 \leq j \leq n}$ be the $2 n \times n$ matrix whose upper $n \times n$ part is $B_{T}$ and whose lower $n \times n$ part is the identity matrix. The matrix $B_{T}$ is skew-symmetric, and each of its entries $b_{i j}$ is either $0,1,-1,2$, or -2 , since every arc $\tau$ can be in at most two triangles. An example where $b_{i j}=2$ is given in Fig. 1 . 
Fig. 1 A triangulation with $b_{23}=2$

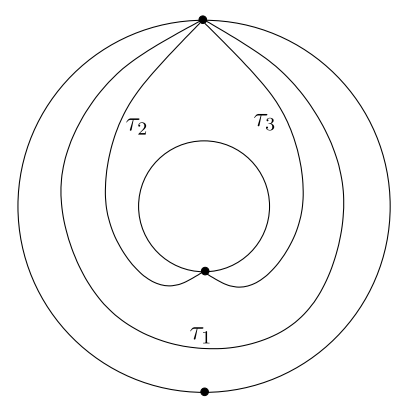

Let $\mathcal{A}\left(\mathbf{x}_{T}, \mathbf{y}_{T}, B_{T}\right)$ be the cluster algebra with principal coefficients for the triangulation $T$, that is, $\mathcal{A}\left(\mathbf{x}_{T}, \mathbf{y}_{T}, B_{T}\right)$ is given by the seed $\left(\mathbf{x}_{T}, \mathbf{y}_{T}, B_{T}\right)$ where $\mathbf{x}_{T}=\left\{x_{\tau_{1}}, x_{\tau_{2}}, \ldots, x_{\tau_{n}}\right\}$ is the cluster associated to the triangulation $T$, and the initial coefficient vector $\mathbf{y}_{T}=\left(y_{1}, y_{2}, \ldots, y_{n}\right)$ is the vector of generators of $\mathbb{P}=$ $\operatorname{Trop}\left(y_{1}, y_{2}, \ldots, y_{n}\right)$. We refer to [18, Definition 2.2] for the definition of tropical semifield.

For the boundary arcs, we define $x_{\tau_{k}}=1, k=n+1, n+2, \ldots, n+m$.

For each $k=1,2, \ldots, n$, there is a unique quadrilateral in $T \backslash\left\{\tau_{k}\right\}$ in which $\tau_{k}$ is one of the diagonals. Let $\tau_{k}^{\prime}$ denote the other diagonal in that quadrilateral. Define the flip $\mu_{k} T$ to be the triangulation $\left(T \backslash\left\{\tau_{k}\right\}\right) \cup\left\{\tau_{k}^{\prime}\right\}$. The mutation $\mu_{k}$ of the seed $\Sigma_{T}$ in the cluster algebra $\mathcal{A}$ corresponds to the flip $\mu_{k}$ of the triangulation $T$ in the following sense: The matrix $\mu_{k}\left(B_{T}\right)$ is the matrix corresponding to the triangulation $\mu_{k} T$, the cluster $\mu_{k}\left(\mathbf{x}_{T}\right)$ is $\left(\mathbf{x}_{T} \backslash\left\{x_{\tau_{k}}\right\}\right) \cup\left\{x_{\tau_{k}^{\prime}}\right\}$, and the corresponding exchange relation is given by

$$
x_{\tau_{k}} x_{\tau_{k}^{\prime}}=x_{\rho_{1}} x_{\rho_{2}} y^{+}+x_{\sigma_{1}} x_{\sigma_{2}} y^{-},
$$

where $y^{+}, y^{-} \in \mathbb{P}$ are some coefficients, and $\rho_{1}, \sigma_{1}, \rho_{2}, \sigma_{2}$ are the sides of the quadrilateral in which $\tau_{k}$ and $\tau_{k}^{\prime}$ are the diagonals, with $\rho_{1}$ opposite to $\rho_{2}$, and $\sigma_{1}$ opposite to $\sigma_{2}$, see [20].

For convenience, we recall the definition of mutation in the cluster algebra. We use the notation $[i]_{+}=\max (i, 0),[1, n]=\{1, \ldots, n\}$, and

$$
\operatorname{sgn}(i)= \begin{cases}-1 & \text { if } i<0 \\ 0 & \text { if } i=0 \\ 1 & \text { if } i>0\end{cases}
$$

Let $\oplus$ denote the addition in $\mathbb{P}$.

Definition 2 (Seed mutations) Let $(\mathbf{x}, \mathbf{y}, B)$ be a seed, and let $k \in[1, n]$. The seed mutation $\mu_{k}$ in direction $k$ transforms $(\mathbf{x}, \mathbf{y}, B)$ into the seed $\mu_{k}(\mathbf{x}, \mathbf{y}, B)=\left(\mathbf{x}^{\prime}, \mathbf{y}^{\prime}, B^{\prime}\right)$ defined as follows:

- The entries of $B^{\prime}=\left(b_{i j}^{\prime}\right)$ are given by

$$
b_{i j}^{\prime}= \begin{cases}-b_{i j} & \text { if } i=k \text { or } j=k \\ b_{i j}+\operatorname{sgn}\left(b_{i k}\right)\left[b_{i k} b_{k j}\right]_{+} & \text {otherwise. }\end{cases}
$$


- The coefficient tuple $\mathbf{y}^{\prime}=\left(y_{1}^{\prime}, \ldots, y_{n}^{\prime}\right)$ is given by

$$
y_{j}^{\prime}= \begin{cases}y_{k}^{-1} & \text { if } j=k \\ y_{j} y_{k}^{\left[b_{k j}\right]_{+}}\left(y_{k} \oplus 1\right)^{-b_{k j}} & \text { if } j \neq k\end{cases}
$$

- The cluster $\mathbf{x}^{\prime}=\left(x_{1}^{\prime}, \ldots, x_{n}^{\prime}\right)$ is given by $x_{j}^{\prime}=x_{j}$ for $j \neq k$, whereas $x_{k}^{\prime}$ is determined by the exchange relation

$$
x_{k}^{\prime}=\frac{y_{k} \prod x_{i}^{\left[b_{i k}\right]_{+}}+\prod x_{i}^{\left[-b_{i k}\right]_{+}}}{\left(y_{k} \oplus 1\right) x_{k}} .
$$

\section{Expansion formula}

In this section, we will present an expansion formula for the cluster variables in terms of perfect matchings of a graph that is constructed recursively using so-called tiles.

\subsection{Tiles}

For the purpose of this paper, a tile $\bar{S}_{k}$ is a planar four-vertex graph with five weighted edges having the shape of two equilateral triangles that share one edge, see Fig. 2. The weight on each edge of the tile $\bar{S}_{k}$ is a cluster variable. The unique interior edge is called diagonal, and the four exterior edges are called sides of $\bar{S}_{k}$. We shall use $S_{k}$ to denote the graph obtained from $\bar{S}_{k}$ by removing the diagonal.

Now let $T$ be a triangulation of the unpunctured surface $(S, M)$. If $\tau_{k} \in T$ is an interior arc, then $\tau_{k}$ lies in precisely two triangles in $T$, hence $\tau_{k}$ is the diagonal of a unique quadrilateral $Q_{\tau_{k}}$ in $T$. We associate to this quadrilateral a tile $\bar{S}_{k}$ by assigning the weight $x_{k}$ to the diagonal and the weights $x_{a}, x_{b}, x_{c}, x_{d}$ to the sides of $\bar{S}_{k}$ in such a way that there is a surjective map $\phi_{k}: Q_{\tau_{k}} \rightarrow \bar{S}_{k}$ which restricts to a homeomorphism between the respective interiors and which sends the arc labeled $\tau_{i}, i=a, b, c, d, k$ to the edge with weight $x_{i}$, see Fig. 2. If $k=1$, we require that $\phi_{1}$ is such that its restriction to the interior is an orientation-preserving homeomorphism, but for $k>1$, we allow the restriction of $\phi_{k}$ to be any homeomorphism.

3.2 The graph $\bar{G}_{T, \gamma}$

Let $T$ be a triangulation of an unpunctured surface $(S, M)$, and let $\gamma$ be an arc in $(S, M)$ which is not in $T$. If necessary, replace $\gamma$ with an isotopic arc so that $\gamma$ intersects transversally each of the arcs in $T$ and minimizes the number of crossings

Fig. 2 The tile $\bar{S}_{k}$

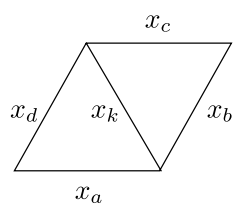


Fig. 3 Glueing tiles $S_{k}$ and $S_{k+1}$ along the edge weighted $x_{\left[\gamma_{k}\right]}$

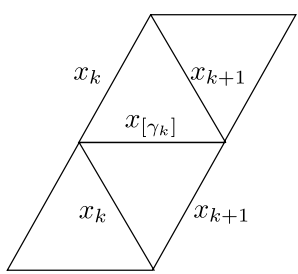

with each of these arcs. An example is given in Fig. 9. Choose an orientation on $\gamma$ and let $s \in M$ be its starting point, and let $t \in M$ be its endpoint. We denote by

$$
p_{0}=s, p_{1}, p_{2}, \ldots, p_{d+1}=t
$$

the points of intersection of $\gamma$ and $T$ in order along $\gamma$ under the orientation chosen above. Let $i_{1}, i_{2}, \ldots, i_{d}$ be such that $p_{k}$ lies on the arc $\tau_{i_{k}} \in T$. Note that $i_{k}$ may be equal to $i_{j}$ even if $k \neq j$. In the example in Sect. 7, this sequence is $i_{1}, i_{2}, i_{3}, i_{4}, i_{1}, i_{2}$. Let $\tilde{S}_{1}, \tilde{S}_{2}, \ldots, \tilde{S}_{d}$ be a sequence of tiles so that $\tilde{S}_{k}$ is isomorphic to the tile $\bar{S}_{i_{k}}$ for $k=1,2, \ldots, d$. In the example in Sect. 7, this sequence is $\bar{S}_{1}, \bar{S}_{2}, \bar{S}_{3}, \bar{S}_{4}, \bar{S}_{1}, \bar{S}_{2}$.

For $k$ from 0 to $d$, let $\gamma_{k}$ denote the segment of the path $\gamma$ from the point $p_{k}$ to the point $p_{k+1}$. Each $\gamma_{k}$ lies in exactly one triangle $\Delta_{k}$ in $T$, and if $1 \leq k \leq d-1$, then $\Delta_{k}$ is formed by the arcs $\tau_{i_{k}}, \tau_{i_{k+1}}$, and a third arc that we denote by $\tau_{\left[\gamma_{k}\right]}$. Note that the arc $\tau_{\left[\gamma_{k}\right]}$ may be a boundary arc. In the example in Sect. 7, the triangle $\Delta_{0}$ has sides $\tau_{5}, \gamma_{1}$, and $\tau_{4}$; the triangle $\Delta_{1}$ has sides $\gamma_{2}, \gamma_{1}$, and $\tau_{6}$.

We will define a graph $\bar{G}_{T, \gamma}$ by recursive glueing of tiles. Start with $\bar{G}_{T, \gamma, 1} \cong \tilde{S}_{1}$, where, if necessary, we rotate the tile $\tilde{S}_{1}$ so that the diagonal goes from northwest to southeast, and the starting point $p_{0}$ of $\gamma$ is in the southwest corner of $\tilde{S}_{1}$. For all $k=1,2, \ldots, d-1$, let $\bar{G}_{T, \gamma, k+1}$ be the graph obtained by adjoining the tile $\tilde{S}_{k+1}$ to the tile $\tilde{S}_{k}$ of the graph $\bar{G}_{T, \gamma, k}$ along the edge weighted $x_{\left[\gamma_{k}\right]}$, see Fig. 3. We always orient the tiles so that the diagonals go from northwest to southeast. This implies that the tiles in odd positions have the orientation induced from the surface and the tiles in even positions have the opposite orientation. Note that the edge weighted $x_{\left[\gamma_{k}\right]}$ is either the northern or the eastern edge of the tile $\tilde{S}_{k}$.

Finally, we define $\bar{G}_{T, \gamma}$ to be $\bar{G}_{T, \gamma, d}$.

Let $G_{T, \gamma}$ be the graph obtained from $\bar{G}_{T, \gamma}$ by removing the diagonal in each tile, that is, $G_{T, \gamma}$ is constructed in the same way as $\bar{G}_{T, \gamma}$ but using the graphs $S_{i_{k}}$ instead of $\bar{S}_{i_{k}}$. For an example see, Fig. 10.

A perfect matching of a graph is a subset of the edges so that each vertex is covered exactly once by an edge in the perfect matching. We define the weight $w(P)$ of a perfect matching $P$ of $G_{T, \gamma}$ to be the product of the weights of all edges in $P$.

\subsection{Cluster expansion formula}

Let $(S, M)$ be an unpunctured surface with triangulation $T$, and let $\mathcal{A}=\mathcal{A}\left(\mathbf{x}_{T}, \mathbf{y}_{T}, B\right)$ be the cluster algebra with principal coefficients in the initial seed $\left(\mathbf{x}_{T}, \mathbf{y}_{T}, B\right)$ defined in Sect. 2. Take an arbitrary cluster variable in $\mathcal{A}$ that is not in the initial cluster $\mathbf{x}$. Since each cluster variable in $\mathcal{A}$ corresponds to an $\operatorname{arc}$ in $(S, M)$, we can denote our 
cluster variable by $x_{\gamma}$ where $\gamma$ is an arc not in $T$. Choose an orientation of $\gamma$, and let $\tau_{i_{1}}, \tau_{i_{2}}, \ldots, \tau_{i_{d}}$ be the arcs of the triangulation that are crossed by $\gamma$ in this order, with multiplicities possible. Let $G_{T, \gamma}$ be the graph constructed in Sect. 3.2.

Theorem 3.1 With the above notation,

$$
x_{\gamma}=\sum_{P} \frac{w(P) y(P)}{x_{i_{1}} x_{i_{2}} \ldots x_{i_{d}}},
$$

where the sum is over all perfect matchings $P$ of $G_{T, \gamma}, w(P)$ is the weight of $P$, and $y(P)$ is a monomial in $\mathbf{y}_{T}$.

The proof of Theorem 3.1 will be given in Sect. 4 .

\section{Proof of Theorem 3.1}

We will use results of [32] to prove the theorem. Throughout this section, $T$ is a triangulation of an unpunctured surface $(S, M), \gamma$ is an arc in $S$ with a fixed orientation, and $s \in M$ is its starting point and $t \in M$ is its endpoint. Moreover, $p_{0}=s, p_{1}, p_{2}, \ldots, p_{d+1}=t$ are the points of intersection of $\gamma$ and $T$ in order along $\gamma$ under the orientation chosen above, and $i_{1}, i_{2}, \ldots, i_{d}$ are such that $p_{k}$ lies on the arc $\tau_{i_{k}} \in T$. Let $\gamma_{k}$ denote the segment of $\gamma$ between the points $p_{k}, p_{k+1}$.

\subsection{Complete $(T, \gamma)$-paths}

Following [33], we will consider paths $\alpha$ in $S$ that are concatenations of arcs and boundary arcs in the triangulation $T$, more precisely, $\alpha=\left(\alpha_{1}, \alpha_{2}, \ldots, \alpha_{\ell(\alpha)}\right)$ with $\alpha_{i} \in T$ for $i=1,2, \ldots, \ell(\alpha)$, and the starting point of $\alpha_{i}$ is the endpoint of $\alpha_{i-1}$. Such a path is called a $T$-path.

We call a $T$-path $\alpha=\left(\alpha_{1}, \alpha_{2}, \ldots, \alpha_{\ell(\alpha)}\right)$ a complete $(T, \gamma)$-path if the following axioms hold:

(T1) The even arcs are precisely the arcs crossed by $\gamma$ in order, that is, $\alpha_{2 k}=\tau_{i_{k}}$.

(T2) For all $k=0,1,2, \ldots, d$, the segment $\gamma_{k}$ is homotopic to the segment of the path $\alpha$ that starts at the point $p_{k}$, then goes along $\alpha_{2 k}$ to the starting point of $\alpha_{2 k+1}$, then along $\alpha_{2 k+1}$ to the starting point of $\alpha_{2 k+2}$, and then along $\alpha_{2 k+2}$ until the point $p_{k+1}$.

We define the Laurent monomial $x(\alpha)$ of the complete $(T, \gamma)$-path $\alpha$ by

$$
x(\alpha)=\prod_{i \text { odd }} x_{\alpha_{i}} \prod_{i \text { even }} x_{\alpha_{i}}^{-1} .
$$

\section{Remark 4.1}

- Every complete $(T, \gamma)$-path starts and ends at the same points as $\gamma$, because of (T2). 
- Every complete $(T, \gamma)$-path has length $2 d+1$.

- For all arcs $\tau$ in the triangulation $T$, the number of times that $\tau$ occurs as $\alpha_{2 k}$ is exactly the number of crossings between $\gamma$ and $\tau$.

- In contrast to the ordinary $(T, \gamma)$-paths defined in [33], complete $(T, \gamma)$-paths allow backtracking.

- The denominator of the Laurent monomial $x(\alpha)$ is equal to $x_{i_{1}} x_{i_{2}} \cdots x_{i_{d}}$.

Example 4.2 The following are two examples of complete $(T, \gamma)$-paths, in the situation in Fig. 9.

$$
\begin{aligned}
& \left(\tau_{5}, \tau_{1}, \tau_{2}, \tau_{2}, \tau_{2}, \tau_{3}, \tau_{7}, \tau_{4}, \tau_{5}, \tau_{1}, \tau_{2}, \tau_{2}, \tau_{8}\right), \\
& \left(\tau_{4}, \tau_{1}, \tau_{1}, \tau_{2}, \tau_{3}, \tau_{3}, \tau_{4}, \tau_{4}, \tau_{5}, \tau_{1}, \tau_{2}, \tau_{2}, \tau_{8}\right) .
\end{aligned}
$$

\subsection{Universal cover}

Let $\pi: \tilde{S} \rightarrow S$ be a universal cover of the surface $S$, and let $\tilde{M}=\pi^{-1}(M)$ and $\tilde{T}=$ $\pi^{-1}(T)$.

Choose $\tilde{s} \in \pi^{-1}(s)$. There exists a unique lift $\tilde{\gamma}$ of $\gamma$ starting at $\tilde{s}$. Then $\tilde{\gamma}$ is the concatenation of subpaths $\tilde{\gamma}_{0}, \tilde{\gamma}_{1}, \ldots, \tilde{\gamma}_{d+1}$ where $\tilde{\gamma}_{k}$ is a path from a point $\tilde{p}_{k}$ to a point $\tilde{p}_{k+1}$ such that $\tilde{\gamma}_{k}$ is a lift of $\gamma_{k}$ and $\tilde{p}_{k} \in \pi^{-1}\left(p_{k}\right)$ for $k=0,1, \ldots, d+1$. Let $\tilde{t}=\tilde{p}_{d+1} \in \pi^{-1}(t)$.

For $k$ from 1 to $d$, let $\tilde{\tau}_{i_{k}}$ be the unique lift of $\tau_{i_{k}}$ running through $\tilde{p}_{k}$, and let $\tilde{\tau}_{\left[\gamma_{k}\right]}$ be the unique lift of $\tau_{\left[\gamma_{k}\right]}$ that is bounding a triangle in $\tilde{S}$ with $\tilde{\tau}_{i_{k}}$ and $\tilde{\tau}_{i_{k+1}}$. Each $\tilde{\gamma}_{k}$ lies in exactly one triangle $\tilde{\Delta}_{k}$ in $\tilde{T}$. Let $\tilde{S}(\gamma) \subset \tilde{S}$ be the union of the triangles $\tilde{\Delta}_{0}, \tilde{\Delta}_{1}, \ldots, \tilde{\Delta}_{d+1}$, and let $\tilde{M}(\gamma)=\tilde{M} \cap \tilde{S}(\gamma)$ and $\tilde{T}(\gamma)=\tilde{T} \cap \tilde{S}(\gamma)$. Then $(\tilde{S}(\gamma), \tilde{M}(\gamma))$ is a simply connected unpunctured surface of which $\tilde{T}(\gamma)$ is a triangulation. This triangulation $\tilde{T}(\gamma)$ consists of arcs, respectively boundary $\operatorname{arcs}, \tilde{\tau}_{i_{k}}, \tilde{\tau}_{\left[\gamma_{k}\right]}$ with $k=1,2, \ldots, d$, and two boundary arcs incident to $\tilde{s}$ and two boundary arcs incident to $\tilde{t}$. The simple connectedness of $\tilde{S}(\gamma)$ follows from the simple connectedness of the universal cover and the fact that the vertices of each triangle lie on the boundary of the universal cover. The fact that $\tilde{T}(\gamma)$ is a triangulation follows from the homotopy lifting property of $\tilde{S}$. Moreover, this triangulation does not contain any internal triangles, since each $\tilde{\tau}_{\left[\gamma_{k}\right]}$ is a boundary arc.

The underlying graph of $\tilde{T}(\gamma)$ is the graph with vertex set $\tilde{M}(\gamma)$ and whose set of edges consists of the (unoriented) arcs in $\tilde{T}(\gamma)$.

By [32, Sect. 5.5], we can compute the Laurent expansion of $x_{\gamma}$ using complete $(\tilde{T}(\gamma), \tilde{\gamma})$-paths in $(\tilde{S}(\gamma), \tilde{M}(\gamma))$.

\subsection{Folding}

The graph $\bar{G}_{T, \gamma}$ was constructed by glueing tiles $\tilde{S}_{k+1}$ to tiles $\tilde{S}_{k}$ along edges with weight $x_{\left[\gamma_{k}\right]}$, see Fig. 3. Now we will fold the graph along the edges weighted $x_{\left[\gamma_{k}\right]}$, thereby identifying the two triangles incident to $x_{\left[\gamma_{k}\right]}, k=1,2, \ldots, d-1$.

To be more precise, the edge with weight $x_{\left[\gamma_{k}\right]}$, that lies in the two tiles $\tilde{S}_{k+1}$ and $\tilde{S}_{k}$, is contained in precisely two triangles $\Delta_{k}$ and $\Delta_{k}^{\prime}$ in $\bar{G}_{T, \gamma}: \Delta_{k}$ lying inside the 
tile $\tilde{S}_{k}$ and $\Delta_{k}^{\prime}$ lying inside the tile $\tilde{S}_{k+1}$. Both $\Delta_{k}$ and $\Delta_{k}^{\prime}$ have weights $x_{\left[\gamma_{k}\right]}, x_{k}$, $x_{k+1}$, but opposite orientations. Cutting $\bar{G}_{T, \gamma}$ along the edge with weight $x_{\left[\gamma_{k}\right]}$, one obtains two connected components. Let $R_{k}$ be the component that contains the tile $\tilde{S}_{k}$ and $R_{k+1}$ the component that contains $\tilde{S}_{k+1}$.

The folding of the graph $\bar{G}_{T, \gamma}$ along $x_{\left[\gamma_{k}\right]}$ is the graph obtained by flipping $R_{k+1}$ and then glueing it to $R_{k}$ by identifying the two triangles $\Delta_{k}$ and $\Delta_{k}^{\prime}$. In this new graph, we can now fold along any of the edges $x_{\left[\gamma_{\ell}\right]}$ with $k \neq \ell$, by cutting along $x_{\left[\gamma_{\ell}\right]}$, defining subgraphs $R_{k, \ell}$ and $R_{k, \ell+1}$ in a similar way, and then flipping $R_{k, \ell+1}$ and glueing it to $R_{k, \ell}$ by identifying the two triangles $\Delta_{\ell}$ and $\Delta_{\ell^{\prime}}$.

The graph obtained by consecutive folding of $\bar{G}_{T, \gamma}$ along all edges with weight $x_{\left[\gamma_{k}\right]}$ for $k=1,2, \ldots, d-1$, is isomorphic to the underlying graph of the triangulation $\tilde{T}(\gamma)$ of the unpunctured surface $(\tilde{S}(\gamma), \tilde{M}(\gamma))$. Indeed, there clearly is a bijection between the triangles in both graphs, and, in both graphs, the way the triangles are glued together is uniquely determined by $\gamma$.

We obtain a map that we call the folding map

$$
\begin{aligned}
\phi:\left\{\begin{array}{c}
\text { perfect matchings } \\
\text { in } G_{T, \gamma}
\end{array}\right\} & \rightarrow\left\{\begin{array}{c}
\text { complete }(\tilde{T}(\gamma), \tilde{\gamma}) \text {-paths } \\
\text { in }(\tilde{S}(\gamma), \tilde{M}(\gamma))
\end{array}\right\} \\
P & \mapsto \quad \tilde{\alpha}_{P}
\end{aligned}
$$

as follows. First, we associate a path $\alpha_{P}$ in $\bar{G}_{T, \gamma}$ to the matching $P$ as follows. Let $\alpha_{P}$ be the path starting at $s$ going along the unique edge of $P$ that is incident to $s$, then going along the diagonal of the first tile $\tilde{S}_{1}$, then along the unique edge of $P$ that is incident to the endpoint of that diagonal, and so forth. The fact that $P$ is a perfect matching guarantees that each endpoint of a diagonal is incident to a unique edge in $P$, and from the construction of $G_{T, \gamma}$ it follows that each edge in $P$ connects two endpoints of two distinct diagonals. It is clear from the construction of $G_{T, \gamma}$ that one can never come back to the same vertex, and therefore the path must reach $t$.

Since $P$ has cardinality $d+1$, the path $\alpha_{P}$ consists of $2 d+1$ edges, thus $\alpha=$ $\left(\alpha_{1}, \alpha_{2}, \ldots, \alpha_{2 d+1}\right)$. Now we define $\tilde{\alpha}_{P}=\left(\tilde{\alpha}_{1}, \tilde{\alpha}_{2}, \ldots, \tilde{\alpha}_{2 d+1}\right)$ by folding the path $\alpha_{P}$. Thus, if $P=\left\{\beta_{1}, \beta_{3}, \ldots, \beta_{2 d-1}, \beta_{2 d+1}\right\}$, where the edges are ordered according to $\gamma$, then $\phi(P)=\left(\tilde{\alpha}_{1}, \tilde{\alpha}_{2}, \ldots, \tilde{\alpha}_{2 d+1}\right)$, where $\tilde{\alpha}_{2 k+1}$ is the image of $\beta_{2 k+1}$ under the folding, and $\tilde{\alpha}_{2 k}=\tilde{\tau}_{i_{k}}$ is the arc crossing $\tilde{\gamma}$ at $\tilde{p}_{k}$. Then $\phi(P)$ satisfies the axiom (T1) by construction. Moreover, $\phi(P)$ satisfies the axiom (T2), because, for each $k=0,1, \ldots, d$, the segment of the path $\phi(P)$, which starts at the point $\tilde{p}_{k}$, then goes along $\tilde{\alpha}_{2 k}$ to the starting point of $\tilde{\alpha}_{2 k+1}$, then along $\tilde{\alpha}_{2 k+1}$ to the starting point of $\tilde{\alpha}_{2 k+2}$, and then along $\tilde{\alpha}_{2 k+2}$ until the point $\tilde{p}_{k+1}$, is homotopic to the segment $\tilde{\gamma}_{k}$, since both segments lie in the simply connected triangle $\tilde{\Delta}_{k}$ formed by $\tilde{\tau}_{i_{k}}, \tilde{\tau}_{i_{k+1}}$, and $\tilde{\tau}_{\left[\gamma_{k}\right]}$. Therefore, the folding map $\phi$ is well defined.

Note that it is possible that $\tilde{\alpha}_{k}, \tilde{\alpha}_{k+1}$ is backtracking, that is, $\tilde{\alpha}_{k}$ and $\tilde{\alpha}_{k+1}$ run along the same arc $\tilde{\tau} \in \tilde{T}(\gamma)$.

Example 4.3 Figure 4 displays an example of a perfect matching $P$, whose edges are the solid bold lines, of the graph $G_{T, \gamma}$ of Fig. 10. The matching contains edges labeled $x_{5}, x_{2}, x_{8}, x_{4}, x_{4}, x_{1}, x_{3}$. The figure also shows the corresponding (not yet 
Fig. 4 Example of complete $(T, \gamma)$ path associated to a matching

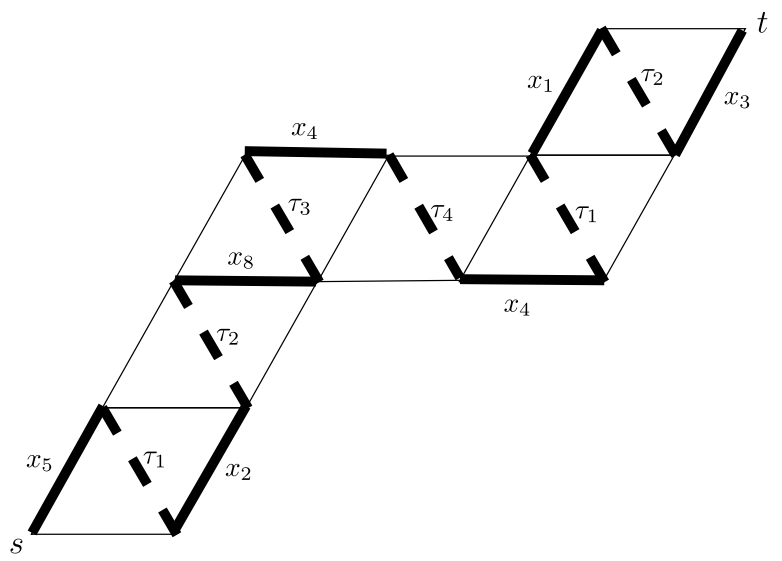

folded) complete $(T, \gamma)$-path obtained by inserting the diagonals $\tau_{1}, \tau_{2}, \tau_{3}, \tau_{4}, \tau_{1}, \tau_{2}$, given as dashed bold lines. In the surface in Fig. 9, the corresponding complete $(T, \gamma)$-path

$$
\alpha_{P}=\left(\tau_{5}, \tau_{1}, \tau_{2}, \tau_{2}, \tau_{8}, \tau_{3}, \tau_{4}, \tau_{4}, \tau_{4}, \tau_{1}, \tau_{1}, \tau_{2}, \tau_{3}\right)
$$

is obtained by folding the path in Fig. 4 .

\subsection{Unfolding the surface}

Let $\alpha$ be a boundary arc in $(\tilde{S}(\gamma), \tilde{M}(\gamma))$ that is not adjacent to $\tilde{s}$ and not adjacent to $\tilde{t}$. Then there is a unique triangle $\Delta$ in $\tilde{T}(\gamma)$ in which $\alpha$ is a side. The other two sides of $\Delta$ are two consecutive arcs, which we denote by $\tilde{\tau}_{j}$ and $\tilde{\tau}_{j+1}$, see Fig. 5 .

By cutting the underlying graph of $\tilde{T}(\gamma)$ along $\tilde{\tau}_{j}$, we obtain two pieces. Let $R_{j+1}$ denote the piece that contains $\alpha, \tilde{\tau}_{j+1}$ and $t$. Similarly, cutting $(\tilde{S}(\gamma), \tilde{M}(\gamma))$ along $\tilde{\tau}_{j+1}$, we obtain two pieces, and we denote by $R_{j}$ the piece that contains $s, \tilde{\tau}_{j}$, and $\alpha$.

The graph obtained by unfolding along $\alpha$ is the graph obtained by flipping $R_{j}$ and then glueing it to $R_{j+1}$ along $\alpha$. In this new graph, we label the edge of $R_{j}$ that had the label $\tilde{\tau}_{j+1}$ by $\tilde{\tau}_{j+1}^{b}$ and the edge of $R_{j+1}$ that had the label $\tilde{\tau}_{j}$ by $\tilde{\tau}_{j}^{b}$, indicating that these edges are on the boundary of the new graph, see Fig. 5. Now, in the graph obtained from unfolding along $\alpha$, we can continue unfolding along (the image of) a different boundary $\operatorname{arc} \alpha^{\prime}$ in $(\tilde{S}(\gamma), \tilde{M}(\gamma))$ that is not adjacent to $\tilde{s}$ and not adjacent to $\tilde{t}$, again using the unique triangle $\Delta^{\prime}$ in $\tilde{T}(\gamma)$ in which $\alpha^{\prime}$ is a side, cutting the graph obtained from unfolding along $\alpha$ along $\tilde{\tau}_{j^{\prime}}$ to obtain $R_{j^{\prime}+1}$ and cutting the graph obtained from unfolding along $\alpha$ along $\tilde{\tau}_{j^{\prime}+1}$ to obtain $R_{j^{\prime}}$, then flipping and glueing in a similar way will give a new graph obtained from $\tilde{T}(\gamma)$ by consecutive unfolding along $\alpha$ and $\alpha^{\prime}$.

Lemma 4.4 The graph obtained by repeated unfolding of the underlying graph of $\tilde{T}(\gamma)$ along all boundary edges not adjacent to $s$ or $t$ is isomorphic to the graph $\bar{G}_{T, \gamma}$. Moreover, for each unfolding along an edge $\alpha$, the edges labeled $\tilde{\tau}_{j}^{b}, \tilde{\tau}_{j+1}^{b}$ are on the boundary of $\bar{G}_{T, \gamma}$ and carry the weights $x_{j}, x_{j+1}$, respectively, the edges 

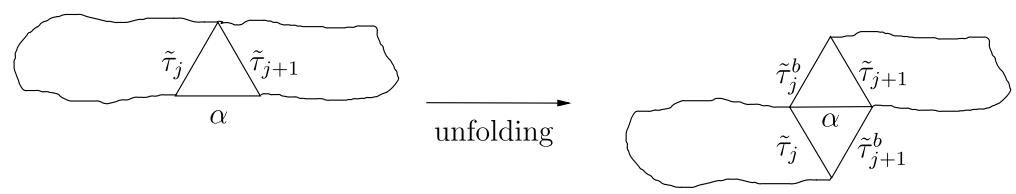

Fig. 5 Completion of paths

labeled $\tilde{\tau}_{j}, \tilde{\tau}_{j+1}$ are diagonals in $\bar{G}_{T, \gamma}$ and carry the weights $x_{j}, x_{j+1}$, respectively, and $\alpha$ is an interior edge of $\bar{G}_{\gamma}$ that is not a diagonal and carries the weight $x_{\left[\gamma_{j}\right]}$.

Proof This follows from the construction.

\subsection{Unfolding map}

We define the map

$$
\begin{aligned}
\{\text { complete }(\tilde{T}(\gamma), \tilde{\gamma})-\text { paths }\} & \left.\rightarrow \text { \{perfect matchings of } G_{T, \gamma}\right\} \\
\tilde{\alpha}=\left(\tilde{\alpha}_{1}, \tilde{\alpha}_{2}, \ldots, \tilde{\alpha}_{2 d+1}\right) & \mapsto P_{\tilde{\alpha}}=\left\{\beta_{1}, \beta_{3}, \beta_{5}, \ldots, \beta_{2 d+1}\right\}
\end{aligned}
$$

where $\beta_{1}=\tilde{\alpha}_{1}, \beta_{2 d+1}=\tilde{\alpha}_{2 d+1}$, and

$$
\beta_{2 k+1}= \begin{cases}\tilde{\alpha}_{2 k+1} & \text { if } \tilde{\alpha}_{2 k+1} \text { is a boundary arc in } \tilde{T}(\gamma), \\ \tilde{\tau}_{j}^{b} & \text { if } \tilde{\alpha}_{2 k+1}=\tilde{\tau}_{j} \text { is an arc in } \tilde{T}(\gamma) .\end{cases}
$$

We will show that this map is well defined. Suppose $\beta_{2 k+1}$ and $\beta_{2 \ell+1}$ have a common endpoint $x$. Then $\tilde{\alpha}_{2 k+1}$ and $\tilde{\alpha}_{2 \ell+1}$ have a common endpoint $y$ in $(\tilde{S}(\gamma), \tilde{M}(\gamma))$, and the two edges are not separated in the unfolding described in Lemma 4.4. Consequently, there is no triangle in $\tilde{T}(\gamma)$ that is contained in the subpolygon spanned by $\tilde{\alpha}_{2 k+1}$ and $\tilde{\alpha}_{2 \ell+1}$, and hence $\tilde{\alpha}_{2 k+1}$ is equal to $\tilde{\alpha}_{2 l+1}$. This implies that every arc in the subpath $\left(\tilde{\alpha}_{2 k+1}, \tilde{\alpha}_{2 k+2}, \ldots, \tilde{\alpha}_{2 \ell+1}\right)$ is equal to the same arc $\tilde{\tau}_{j}$, and the only way this can happen is when $\ell=k+1$ and $\left(\tilde{\alpha}_{2 k+1}, \tilde{\alpha}_{2 k+2}, \ldots, \tilde{\alpha}_{2 \ell+1}\right)=\left(\tilde{\tau}_{j}, \tilde{\tau}_{j}, \tilde{\tau}_{j}\right)$ and both endpoints of $\tilde{\tau}_{j}$ are incident to an interior arc other than $\tilde{\tau}_{j}$. In this case, $\tilde{\tau}_{j}$ bounds the two triangles $\tilde{\tau}_{j-1}, \tilde{\tau}_{j}, \tilde{\tau}_{\left[\gamma_{j-1}\right]}$ and $\tilde{\tau}_{j}, \tilde{\tau}_{j+1}, \tilde{\tau}_{\left[\gamma_{j}\right]}$ in $\tilde{T}(\gamma)$. Unfolding along $\tilde{\tau}_{\left[\gamma_{j-1}\right]}$ and $\tilde{\tau}_{\left[\gamma_{j}\right]}$ will produce edges $\beta_{2 k+1}$ and $\beta_{2 \ell+1}$ that are not adjacent, see Fig. 6 .

This shows that no vertex of $G_{T, \gamma}$ is covered twice in $P_{\tilde{\alpha}}$.

To show that every vertex of $G_{T, \gamma}$ is covered in $P_{\tilde{\alpha}}$, we use a counting argument. Indeed, the number of vertices of $G_{T, \gamma}$ is $2(d+1)$, and, on the other hand, $2 d+1$ is the length of $\tilde{\alpha}$, since $\tilde{\alpha}$ is complete, and thus $P_{\tilde{\alpha}}$ has $d+1$ edges. The statement follows since every $\beta_{j} \in P_{\tilde{\alpha}}$ has two distinct endpoints. This shows that $P_{\tilde{\alpha}}$ is a perfect matching and our map is well defined.

Lemma 4.5 The unfolding map $\tilde{\alpha} \mapsto P_{\tilde{\alpha}}$ is the inverse of the folding map $P \mapsto \tilde{\alpha}_{P}$. In particular, both maps are bijections.

Proof Let $\tilde{\alpha}=\left(\tilde{\alpha}_{1}, \tilde{\alpha}_{2}, \ldots, \tilde{\alpha}_{2 d+1}\right)$ be a complete $(\tilde{T}(\gamma), \tilde{\gamma})$-path. Then $\tilde{\alpha}_{P_{\tilde{\alpha}}}=\left(\alpha_{1}\right.$, $\left.\alpha_{2}, \ldots, \alpha_{2 d+1}\right)$ where $\alpha_{2 k+1}$ is the image under folding of the arc $\tilde{\tau}_{j}^{b}$ if $\tilde{\alpha}_{2 k+1}=\tilde{\tau}_{j}$ 

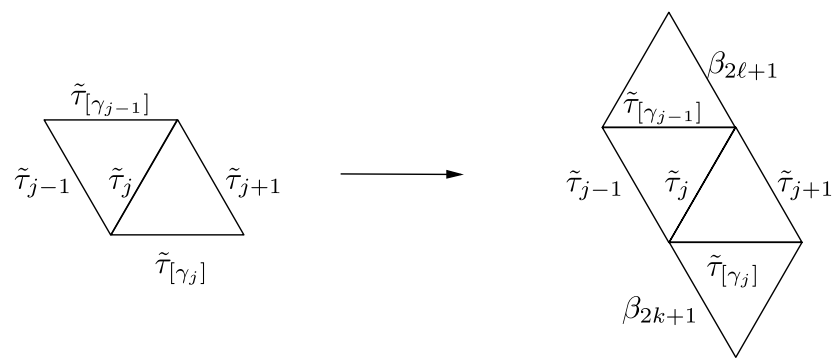

Fig. 6 Unfolding along $\tilde{\tau}_{\left[\gamma_{j-1}\right]}$ and $\tilde{\tau}_{\left[\gamma_{j}\right]}$

is an arc in $\tilde{T}(\gamma)$ or, otherwise, the image under the folding of the arc $\tilde{\alpha}_{2 k+1}$. Thus $\alpha_{2 k+1}=\tilde{\alpha}_{2 k+1}$. Moreover, $\alpha_{2 k}=\tau_{i_{k}}=\tilde{\alpha}_{2 k}$, and thus $\tilde{\alpha}_{P_{\tilde{\alpha}}}=\tilde{\alpha}$.

Conversely, let $P=\left\{\beta_{1}, \beta_{3}, \ldots, \beta_{2 d-1}, \beta_{2 d+1}\right\}$ be a perfect matching of $G_{T, \gamma}$. Then $P_{\tilde{\alpha}_{P}}=\left\{\tilde{\beta}_{1}, \tilde{\beta}_{3}, \ldots, \tilde{\beta}_{2 d-1}, \tilde{\beta}_{2 d+1}\right\}$ where

$$
\begin{aligned}
\tilde{\beta}_{2 k+1} & = \begin{cases}\tilde{\alpha}_{2 k+1} & \text { if } \tilde{\alpha}_{2 k+1} \text { is a boundary arc, } \\
\tilde{\tau}_{j}^{b} & \text { if } \tilde{\alpha}_{2 k+1}=\tilde{\tau}_{j} \text { is an arc }\end{cases} \\
& = \begin{cases}\tilde{\tau}_{\left[\gamma_{j}\right]} & \text { if } \beta_{2 k+1}=\tilde{\tau}_{\left[\gamma_{j}\right]}, \\
\tilde{\tau}_{j}^{b} & \text { if } \beta_{2 k+1}=\tilde{\tau}_{j}^{b} .\end{cases}
\end{aligned}
$$

Hence $P_{\tilde{\alpha}_{P}}=P$.

Combining Lemma 4.5 with the results of [32], we obtain the following theorem.

Theorem 4.6 There is a bijection between the set of perfect matchings of the graph $G_{T, \gamma}$ and the set of complete $(T, \gamma)$-paths in $(S, M)$ given by $P \mapsto \pi\left(\tilde{\alpha}_{P}\right)$, where $\tilde{\alpha}_{P}$ is the image of $P$ under the folding map, and $\pi$ is induced by the universal cover $\pi$ : $\tilde{S} \rightarrow S$. Moreover, the numerator of the Laurent monomial $x\left(\pi\left(\tilde{\alpha}_{P}\right)\right)$ of the complete $(T, \gamma)$-path $\pi\left(\tilde{\alpha}_{P}\right)$ is equal to the weight $w(P)$ of the matching $P$.

Proof The map in the theorem is a bijection, because it is the composition of the folding map, which is a bijection, by Lemma 4.5, and the map $\pi$, which is a bijection, by [32, Lemma 5.8]. The last statement of the theorem follows from the construction of the graph $G_{T, \gamma}$.

Example 4.7 The unfolding of the path

$$
\tilde{\alpha}=\left(\tau_{5}, \tau_{1}, \tau_{2}, \tau_{2}, \tau_{8}, \tau_{3}, \tau_{4}, \tau_{4}, \tau_{4}, \tau_{1}, \tau_{1}, \tau_{2}, \tau_{3}\right)
$$

in the surface of Fig. 9 is the perfect matching $P_{\tilde{\alpha}}=P$ of Example 4.3. 


\subsection{Proof of Theorem 3.1}

It has been shown in [32, Theorem 3.2] that

$$
x_{\gamma}=\sum_{\alpha} x(\alpha) y(\alpha)
$$

where the sum is over all complete $(T, \gamma)$-paths $\alpha$ in $(S, M), y(\alpha)$ is a monomial in $\mathbf{y}_{T}$, and

$$
x(\alpha)=\prod_{k \text { odd }} x_{\alpha_{k}} \prod_{k \text { even }} x_{\alpha_{k}}^{-1} .
$$

Applying Theorem 4.6 to (5) yields

$$
x_{\gamma}=\sum_{P} w(P) y(P)\left(x_{i_{1}} x_{i_{2}} \cdots x_{i_{d}}\right)^{-1}
$$

where the sum is over all perfect matchings $P$ of $G_{T, \gamma}, w(P)$ is the weight of the matching and $y(P)=y\left(\pi\left(\tilde{\alpha}_{P}\right)\right)$, by definition. This completes the proof of Theorem 3.1 .

\section{A formula for $y(P)$}

In this section, we give a description of the coefficients $y(P)$ in terms of the matching $P$. First, we need to recall some results from [32].

Recall that $T$ is a triangulation of the unpunctured surface $(S, M)$ and that $\gamma$ is an arc in $(S, M)$ that crosses $T$ exactly $d$ times. We also have fixed an orientation for $\gamma$ and denote by $s=p_{0}, p_{1}, \ldots, p_{d}, p_{d+1}=t$ the intersection points of $\gamma$ and $T$ in order of occurrence on $\gamma$. Let $i_{1}, i_{2}, \ldots, i_{d}$ be such that $p_{k}$ lies on the arc $\tau_{i_{k}} \in T$ for $k=1,2, \ldots, d$. For $k=0,1, \ldots, d$, let $\gamma_{k}$ denote the segment of the path $\gamma$ from the point $p_{k}$ to the point $p_{k+1}$. Each $\gamma_{k}$ lies in exactly one triangle $\Delta_{k}$ in $T$. If $1 \leq k \leq$ $d-1$, the triangle $\Delta_{k}$ is formed by the arcs $\tau_{i_{k}}, \tau_{i_{k+1}}$ and a third arc that we denote by $\tau_{\left[\gamma_{k}\right]}$.

The orientation of the surface $S$ induces an orientation on each of these triangles in such a way that, whenever two triangles $\Delta, \Delta^{\prime}$ share an edge $\tau$, then the orientation of $\tau$ in $\Delta$ is opposite to the orientation of $\tau$ in $\Delta^{\prime}$, There are precisely two such orientations. We assume without loss of generality that $S$ has the "clockwise orientation," that is, in each triangle $\Delta$, going around the boundary of $\Delta$ according to the orientation of $S$, is clockwise when looking at it from outside the surface.

Let $\alpha$ be a complete $(T, \gamma)$-path. Then $\alpha_{2 k}=\tau_{i_{k}}$ is a common arc of the two triangles $\Delta_{k-1}$ and $\Delta_{k}$. We say that $\alpha_{2 k}$ is $\gamma$-oriented if the orientation of $\alpha_{2 k}$ in the path $\alpha$ is the same as the orientation of $\tau_{i_{k}}$ in the triangle $\Delta_{k}$, see Fig. 7 .

It is shown in [32, Theorem 3.2] that

$$
y(\alpha)=\prod_{k: \alpha_{2 k}} y_{i s} \gamma \text {-oriented }
$$



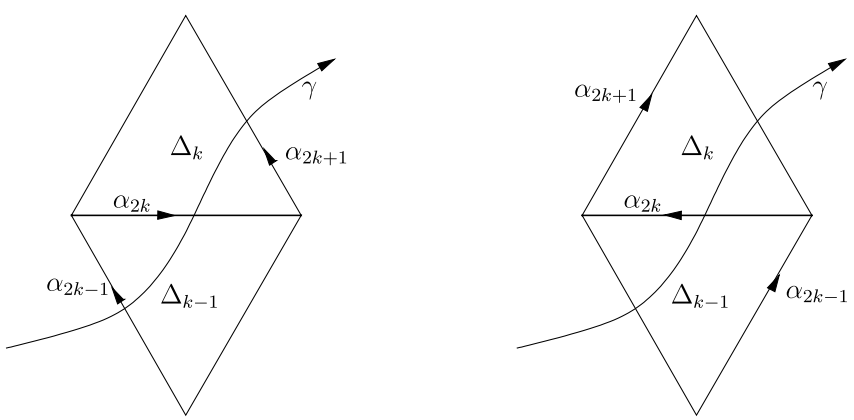

Fig. 7 Two examples of the $(T, \gamma)$-path segment $\left(\alpha_{2 k-1}, \alpha_{2 k}, \alpha_{2 k+1}\right)$. On the left, $\alpha_{2 k}$ is not $\gamma$-oriented, and on the right, $\alpha_{2 k}$ is $\gamma$-oriented

Each perfect matching $P$ of $G_{T, \gamma}$ induces a path $\alpha_{P}$ in $\bar{G}_{T, \gamma}$ as in the construction of the folding map in Sect. 4.3. The even arcs of $\alpha_{P}$ are the diagonals of the graph $\bar{G}_{T, \gamma}$. We say that an even arc of $\alpha_{P}$ has upward orientation if $\alpha_{P}$ is directed from southeast to northwest on that even arc, otherwise we say that the arc has downward orientation. Since going upward on the first even arc of $\alpha_{P}$ is $\gamma$-oriented, we have that the (2k)th arc of $\pi\left(\tilde{\alpha}_{P}\right)$ is $\gamma$-oriented if and only if the (2k)th arc of $\alpha_{P}$ is upward if $k$ is odd and downward if $k$ is even.

There are precisely two perfect matchings $P_{+}$and $P_{-}$of $G_{T, \gamma}$ that contain only boundary edges of $G_{T, \gamma}$. The orientations of the even arcs in both of the induced $(T, \gamma)$-paths $\tilde{\alpha}_{P_{+}}$and $\tilde{\alpha}_{P_{-}}$are alternatingly upward and downward, thus for one of the two paths, say $P_{+}$, each even arc of $\pi\left(\tilde{\alpha}_{P_{+}}\right)$is $\gamma$-oriented, whereas for $P_{-}$, none of the even arcs of $\pi\left(\tilde{\alpha}_{P_{-}}\right)$is $\gamma$-oriented. That is, $y\left(P_{-}\right)=1$ and $y\left(P_{+}\right)=y_{i_{1}} y_{i_{2}} \cdots y_{i_{d}}$. Note that, since the tile $\tilde{S}_{1}$ has the same orientation as the surface, the matching $P_{-}$ contains the western edge of $\tilde{S}_{1}$, while $P_{+}$contains the southern edge of $\tilde{S}_{1}$.

For an arbitrary perfect matching $P$, the coefficient $y(P)$ is determined by the set of edges of the symmetric difference $P_{-} \ominus P=\left(P_{-} \cup P\right) \backslash\left(P_{-} \cap P\right)$ as follows.

Theorem 5.1 The set $P_{-} \ominus P$ is the set of boundary edges of a (possibly disconnected) subgraph $G_{P}$ of $G_{T, \gamma}$ which is a union of tiles

$$
G_{P}=\bigcup_{j \in J} S_{j} .
$$

Moreover,

$$
y(P)=\prod_{j \in J} y_{i_{j}} .
$$

Proof Choose any edge $e_{1}$ and either endpoint in $P_{-} \backslash\left(P_{-} \cap P\right)$, and walk along that edge until its other endpoint. Since $P$ is a perfect matching, this endpoint is incident to an edge $e_{2}$ in $P$, which is different from $e_{1}$ and, hence, not in $P_{-}$. Thus $e_{2} \in P \backslash\left(P_{-} \cap P\right)$. Now walk along $e_{2}$ until its other endpoint. This endpoint is incident to an edge $e_{3}$ in $P_{-}$which is different from $e_{2}$ and, hence, not in $P$. Thus 
$e_{3} \in P_{-} \backslash\left(P_{-} \cap P\right)$. Continuing this way, we construct a sequence of edges in $P_{-} \ominus P$. Since $G_{T, \gamma}$ has only finitely many edges, this sequence must become periodic after a certain number of steps; thus there exist $p, N$ such that $e_{k}=e_{k+p}$ for all $k \geq N$.

We will show that one can take $N=1$. Suppose to the contrary that $N \geq 2$ is the smallest integer such that $e_{k}=e_{k+p}$ for all $k \geq N$. Then $e_{N-1}, e_{N}$ and $e_{N+p-1}$ share a common endpoint. But $e_{N-1}, e_{N}$ and $e_{N+p-1}$ are elements of the union of two perfect matchings, hence $e_{N-1}=e_{N+p-1}$, contradicting the minimality of $N$.

Therefore the sequence $e_{1}, e_{2}, \ldots, e_{p}$ in $P \ominus P_{-}$is the set of boundary edges of a connected subgraph of $G_{T, \gamma}$ which is a union of tiles.

The graph $G_{P}$ is the union of these connected subgraphs and, hence, is a union of tiles. Let $H$ be a connected component of $G_{P}$. There are precisely two perfect matchings $P_{-}(H)$ and $P_{+}(H)$ of $H$ that consist only of boundary edges of $H$. Clearly, these two matchings are $P_{-} \cap E(H)$ and $P \cap E(H)$, where $E(H)$ is the set of edges of the graph $H$. Therefore, in each tile of $H$, the orientations of the diagonal in $\alpha_{P_{-}}$and $\alpha_{P}$ are opposite. The restrictions of $P_{-}$and $P$ to $E\left(G_{T, \gamma}\right) \backslash E\left(G_{P}\right)$ are identical, hence in each tile of $G_{T, \gamma} \backslash G_{P}$, the orientations of the diagonal in $\alpha_{P_{-}}$and $\alpha_{P}$ are equal. It follows from (8) that $y(P)=\prod_{j \in J} y_{i_{j}}$.

It has been shown in [18] that, for any cluster variable $x_{\gamma}$ in $\mathcal{A}$, its Laurent expansion in the initial seed $\left(\mathbf{x}_{T}, \mathbf{y}_{T}, B_{T}\right)$ is homogeneous with respect to the grading given by $\operatorname{deg}\left(x_{i}\right)=\mathbf{e}_{i}$ and $\operatorname{deg}\left(y_{i}\right)=B_{T} \mathbf{e}_{i}$, where $\mathbf{e}_{i}=(0, \ldots, 0,1,0, \ldots, 0)^{T} \in \mathbb{Z}^{n}$ with 1 at position $i$. By definition, the $g$-vector $g_{\gamma}$ of a cluster variable $x_{\gamma}$ is the degree of its Laurent expansion with respect to this grading.

Corollary 5.2 The g-vector $g_{\gamma}$ of $x_{\gamma}$ is given by

$$
g_{\gamma}=\operatorname{deg} \frac{w\left(P_{-}\right)}{x_{i_{1}} x_{i_{2}} \cdots x_{i_{d}}} .
$$

Proof This follows from the fact that $y\left(P_{-}\right)=1$.

Remark 5.3 The formula for $y(P)$ can also be phrased in terms of height functions. As described in Sect. 3 of [29], one way to define the height function on the faces of a bipartite planar graph $G$, covered by a perfect matching $P$, is to superimpose each matching with the fixed matching $P_{\hat{0}}$ (the unique matching of minimal height). In the case where $G$ is a snake graph, we take $P_{\hat{0}}$ to be $P_{-}$, the matching of $G$ only involving edges on the boundary and including the western edge of tile $\tilde{S}_{1}$. Color the vertices of $G$ black and white so that no two adjacent vertices have the same color. In this superposition, we orient edges of $P$ from black to white, and edges of $P_{-}$ from white to black. We thereby obtain a spanning set of cycles, and removing the cycles of length two exactly corresponds to taking the symmetric difference $P \ominus P_{-}$. We can read the resulting graph as a relief-map, in which the altitude changes by +1 or -1 as one crosses over a contour line, according to whether the counter-line is directed clockwise or counter-clockwise. By this procedure, we obtain a height function $h_{P}: F(G) \rightarrow \mathbb{Z}$ which assigns integers to the faces of graph $G$. When $G$ is 

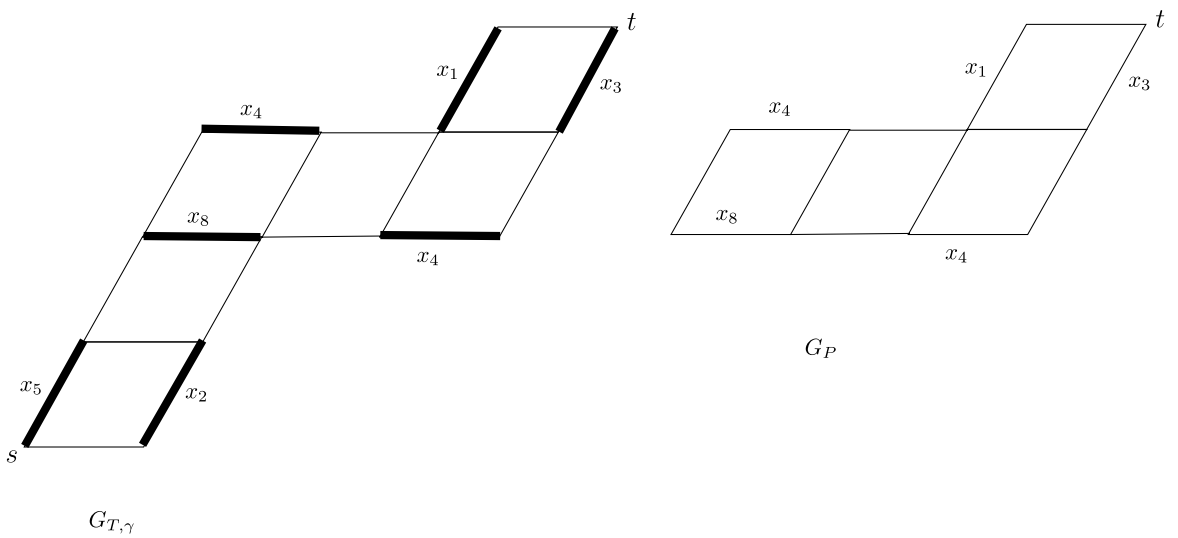

$G_{P}$

Fig. 8 Perfect matching $P$ and associated graph $G_{P}$

a snake graph, the set of faces $F(G)$ is simply the set of tiles $\left\{S_{j}\right\}$ of $G$. Comparing with the definition of $y(P)$ in Theorem 5.1, we see that

$$
y(P)=\prod_{S_{j} \in F(G)} y_{j}^{h_{P}(j)} .
$$

An alternative definition of height functions comes from [12] by translating the matching problem into a domino tiling problem on a region colored as a checkerboard. We imagine an ant starting at an arbitrary vertex at height 0 , walking along the boundary of each domino, and changing its height by +1 or -1 as it traverses the boundary of a black or white square, respectively. The values of the height function under these two formulations agree up to scaling by four.

Example 5.4 We illustrate the construction of the graph $G_{P}$ in Fig. 8. The perfect matching of $G_{T, \gamma}$ is the set of bold face edges on the left side of the figure, and the graph $G_{P}$ is given on the right. Note that the matching $P_{-}$in this example consists of the two edges labeled $x_{2}, x_{5}$ in the first tile and the boundary edges of the graph $G_{P}$ that are not in $G_{P}$.

\section{Cluster expansion without matchings}

In this section, we give a formula for the cluster expansion of $x_{\gamma}$ in terms of the graph $G_{T, \gamma}$ only.

For any subgraph $H$ of $G_{T, \gamma}$, let $c(H)$ be the number of connected components of $H$. Let $E(H)$ be the set of edges of $H$, and denote by $\partial H$ the set of boundary edges of $H$. Define $\mathcal{H}_{k}$ to be the set of all subgraphs $H$ of $G_{T, \gamma}$ such that $H$ is a union of $k$ tiles $H=S_{j_{1}} \cup \cdots \cup S_{j_{k}}$ and the number of edges of $P_{-}$that are contained 
in $H$ is equal to $k+c(H)$. For $H \in \mathcal{H}_{k}$, let

$$
y(H)=\prod_{S_{i_{j}} \text { tile in } H} y_{i_{j}} .
$$

Theorem 6.1 The cluster expansion of the cluster variable $x_{\gamma}$ is given by

$$
x_{\gamma}=\sum_{k=0}^{d} \sum_{H \in \mathcal{H}_{k}} \frac{w\left(\partial H \ominus P_{-}\right) y(H)}{x_{i_{1}} x_{i_{2}} \ldots x_{i_{d}}} .
$$

Proof It follows from Theorems 3.1 and 5.1 that

$$
x_{\gamma}=\sum_{k=1}^{d} \sum_{P:|y(P)|=k} \frac{w(P) y\left(G_{P}\right)}{x_{i_{1}} x_{i_{2}} \ldots x_{i_{d}}},
$$

where $|y(P)|$ is the number of tiles in $G_{P}$. We will show that for all $k$, the map $P \mapsto G_{P}$ is a bijection between the set of perfect matchings $P$ of $G_{T, \gamma}$ such that $|y(P)|=k$ and the set $\mathcal{H}_{k}$.

- The map is well-defined. Clearly, $G_{P}$ is the union of $k$ tiles. Moreover, $E\left(G_{P}\right) \cap$ $P_{-}$is a perfect matching of $G_{P}$, since $P_{-}$consists of every other boundary edge of $G_{T, \gamma}$. Thus the cardinality of $\left(E\left(G_{P}\right) \cap P_{-}\right)$is half the number of vertices of $G_{P}$, which is equal to $2 k+2 c\left(G_{P}\right)$. Therefore, the cardinality of $\left(E\left(G_{P}\right) \cap P_{-}\right)$ is $k+c\left(G_{P}\right)$, and $G_{P} \in \mathcal{H}_{k}$.

- The map is injective, since two graphs $G_{P}, G_{P^{\prime}}$ are equal if and only if their boundaries are.

- The map is surjective. Let $H=S_{j_{1}} \cup \cdots \cup S_{j_{k}}$ be such that the cardinality of $E(H) \cap P_{-}$equals $k+c(H)$. The boundary of $H$ consists of $2 k+2 c(H)$ edges, half of which lie in $P_{-}$. As in the proof of Theorem 5.1, let $P_{-}(H)=E(H) \cap P_{-}$ and $P_{+}(H)$ be the two perfect matchings of $H$ that consist of boundary edges only. Let $P=P_{+}(H) \cup\left(P_{-} \backslash P_{-}(H)\right)$. Then $P$ is a perfect matching of $G_{T, \gamma}$ such that $G_{P}=H$, and moreover, $|y(P)|$ is equal to the number of tiles in $H$, which is $k$. Thus the map is surjective.

Now the boundary edges of $G_{P}$ are precisely the elements of $P \ominus P_{-}$, which implies that $\partial\left(G_{P}\right) \ominus P_{-}=\left(P \ominus P_{-}\right) \ominus P_{-}=P \ominus\left(P_{-} \ominus P_{-}\right)=P$. Therefore $w(P)=w\left(\partial\left(G_{P}\right) \ominus P_{-}\right)$, and this completes the proof.

Corollary 6.2 The F-polynomial of $\gamma$ is given by

$$
F_{\gamma}=\sum_{k=0}^{d} \sum_{H \in \mathcal{H}_{k}} y(H)
$$




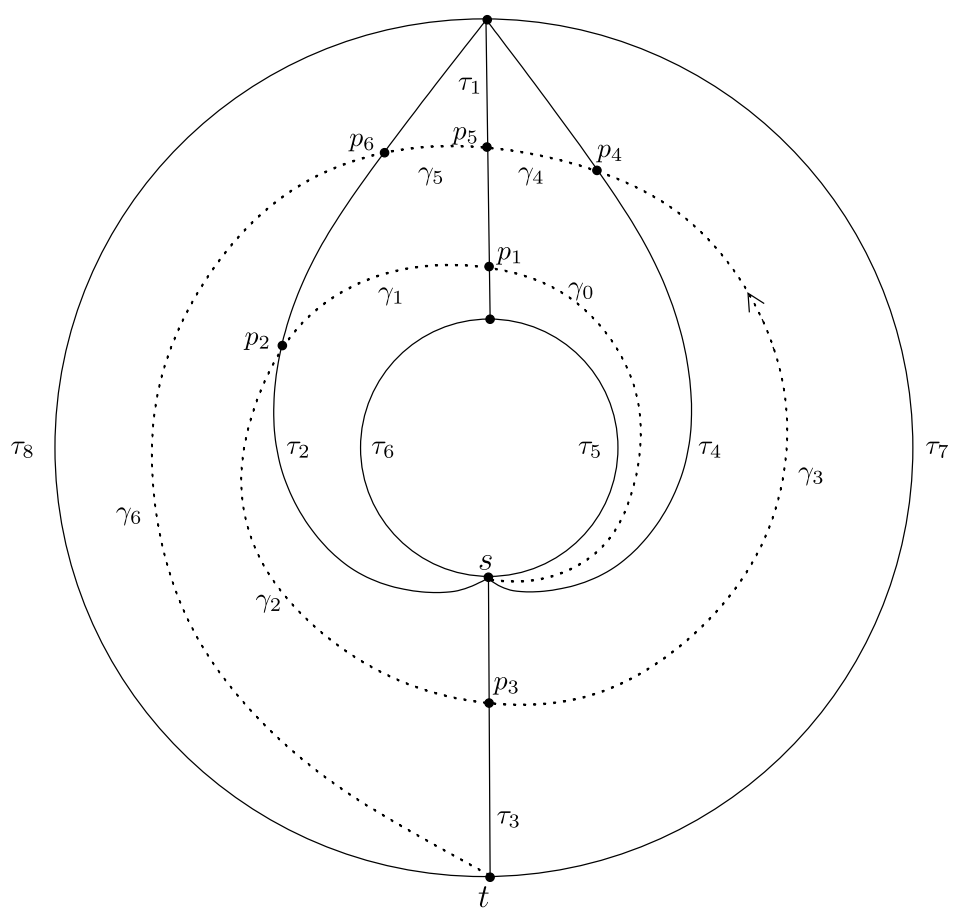

Fig. 9 Triangulated surface with dotted $\operatorname{arc} \gamma$

\section{Example}

We illustrate Theorem 3.1, Theorem 5.1, and Theorem 6.1 in an example. Let $(S, M)$ be the annulus with two marked points on each of the two boundary components, and let $T=\left\{\tau_{1}, \ldots, \tau_{8}\right\}$ be the triangulation shown in Fig. 9. The corresponding cluster algebra has the following principal exchange matrix:

$$
\left[\begin{array}{cccc}
0 & 1 & 0 & -1 \\
-1 & 0 & -1 & 0 \\
0 & 1 & 0 & -1 \\
1 & 0 & 1 & 0
\end{array}\right],
$$

which can also be visualized by a quiver, simply by drawing $b_{i j}$ arrows $i \rightarrow j$ for each positive entry $b_{i j}$ in the matrix:

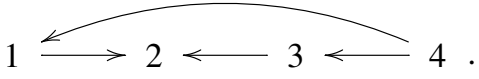

Let $\gamma$ be the dotted arc in Fig. 9. It has $d=6$ crossings with the triangulation. The sequence of crossed arcs $\tau_{i_{1}}, \ldots, \tau_{i_{6}}$ is $\tau_{1}, \tau_{2}, \tau_{3}, \tau_{4}, \tau_{1}, \tau_{2}$, and the corresponding segments $\gamma_{0}, \ldots, \gamma_{6}$ of the arc $\gamma$ are labeled in the figure. Moreover, $\tau_{\left[\gamma_{1}\right]}=\tau_{6}, \tau_{\left[\gamma_{2}\right]}=$ $\tau_{8}, \tau_{\left[\gamma_{3}\right]}=\tau_{7}, \tau_{\left[\gamma_{4}\right]}=\tau_{5}$, and $\tau_{\left[\gamma_{5}\right]}=\tau_{6}$. 

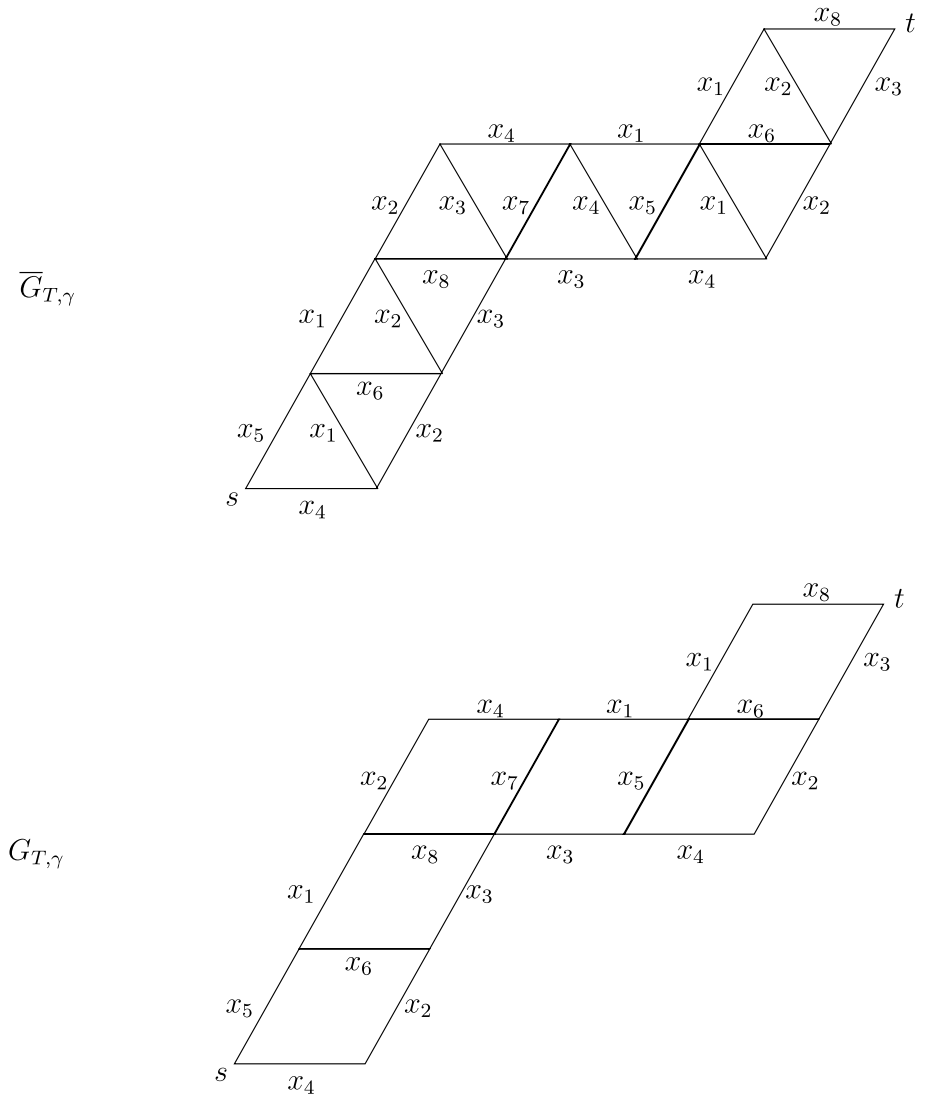

Fig. 10 Construction of the graphs $\bar{G}_{T, \gamma}$ and $G_{T, \gamma}$

The graph $G_{T, \gamma}$ is obtained by glueing the corresponding six tiles $\tilde{S}_{1}, \tilde{S}_{2}, \tilde{S}_{3}, \tilde{S}_{4}$, $\tilde{S}_{1}$, and $\tilde{S}_{2}$. The result is shown in Fig. 10 .

Theorems 3.1 and 5.1 imply that $x_{\gamma}\left(x_{i_{1}} x_{i_{2}} \cdots x_{i_{d}}\right)$ is equal to

$$
\begin{array}{cccc} 
& x_{5} x_{2} x_{2} x_{3} x_{1} x_{2} x_{8} & + & x_{4} x_{6} x_{2} x_{3} x_{1} x_{2} x_{8} y_{1} \\
+ & x_{5} x_{2} x_{2} x_{7} x_{5} x_{2} x_{8} y_{4} & + & x_{4} x_{6} x_{2} x_{7} x_{5} x_{2} x_{8} y_{1} y_{4} \\
+ & x_{5} x_{2} x_{8} x_{4} x_{5} x_{2} x_{8} y_{3} y_{4} & + & x_{5} x_{2} x_{2} x_{7} x_{4} x_{6} x_{8} y_{4} y_{1} \\
+ & x_{4} x_{6} x_{8} x_{4} x_{5} x_{2} x_{8} y_{1} y_{3} y_{4} & + & x_{4} x_{6} x_{2} x_{7} x_{4} x_{6} x_{8} y_{1} y_{4} y_{1} \\
+ & x_{5} x_{2} x_{8} x_{4} x_{4} x_{6} x_{8} y_{3} y_{4} y_{1} & + & x_{5} x_{2} x_{2} x_{7} x_{4} x_{1} x_{3} y_{4} y_{1} y_{2} \\
+ & x_{4} x_{1} x_{3} x_{4} x_{5} x_{2} x_{8} y_{1} y_{2} y_{3} y_{4} & + & x_{4} x_{6} x_{8} x_{4} x_{4} x_{6} x_{8} y_{1} y_{3} y_{4} y_{1} \\
+ & x_{4} x_{6} x_{2} x_{7} x_{4} x_{1} x_{3} y_{1} y_{4} y_{1} y_{2} & + & x_{5} x_{2} x_{8} x_{4} x_{4} x_{1} x_{3} y_{3} y_{4} y_{1} y_{2} \\
+ & x_{4} x_{1} x_{3} x_{4} x_{4} x_{6} x_{8} y_{1} y_{2} y_{3} y_{4} y_{1} & + & x_{4} x_{6} x_{8} x_{4} x_{4} x_{1} x_{3} y_{1} y_{3} y_{4} y_{1} y_{2} \\
+ & x_{4} x_{1} x_{3} x_{4} x_{4} x_{1} x_{3} y_{1} y_{2} y_{3} y_{4} y_{1} y_{2}, & &
\end{array}
$$


which is equal to

$$
\begin{array}{cccc} 
& x_{1} x_{2}^{3} x_{3} & + & x_{1} x_{2}^{2} x_{3} x_{4} y_{1} \\
+ & x_{2}^{3} y_{4} & + & x_{2}^{2} x_{4} y_{1} y_{4} \\
+ & x_{2}^{2} x_{4} y_{3} y_{4} & + & x_{2}^{2} x_{4} y_{1} y_{4} \\
+ & x_{2} x_{4}^{2} y_{1} y_{3} y_{4} & + & x_{2} x_{4}^{2} y_{1}^{2} y_{4} \\
+ & x_{2} x_{4}^{2} y_{3} y_{4} y_{1} & + & x_{1} x_{2}^{2} x_{3} x_{4} y_{1} y_{2} y_{4} \\
+ & x_{1} x_{2} x_{3} x_{4}^{2} y_{1} y_{2} y_{3} y_{4} & + & x_{4}^{3} y_{1}^{2} y_{3} y_{4} \\
+ & x_{1} x_{2} x_{3} x_{4}^{2} y_{1}^{2} y_{2} y_{4} & + & x_{1} x_{2} x_{3} x_{4}^{2} y_{3} y_{4} y_{1} y_{2} \\
+ & x_{1} x_{3} x_{4}^{3} y_{1}^{2} y_{2} y_{3} y_{4} & + & x_{1} x_{3} x_{4}^{3} y_{1}^{2} y_{2} y_{3} y_{4} \\
+ & x_{1}^{2} x_{3}^{2} x_{4}^{2} y_{1}^{2} y_{2}^{2} y_{3} y_{4} . & &
\end{array}
$$

The first term corresponds to the matching $P_{-}$consisting of the boundary edges weighted $x_{5}$ and $x_{2}$ in the first tile, $x_{2}$ in the third tile, $x_{1}$ and $x_{3}$ in the forth, $x_{2}$ in the fifth, and $x_{8}$ in the sixth tile. For example, the twelfth term corresponds to the matching $P$ consisting of the horizontal edges of the first three tiles and the horizontal edges of the last two tiles. Thus $P_{-} \ominus P=\left(P_{-} \cup P\right) \backslash\left(P_{-} \cap P\right)$ is the union of the first, third, forth, and fifth tiles, whence $y(P)=y_{i_{1}} y_{i_{3}} y_{i_{4}} y_{i_{5}}=y_{1} y_{3} y_{4} y_{1}$.

To illustrate Theorem 6.1, let $k=2$. Then $\mathcal{H}_{k}$ consists of the subgraphs $H$ of $G_{T, \gamma}$ which are unions of two tiles and such that $E(H) \cap P_{-}$has three elements if $H$ is connected, respectively four elements if $H$ has two connected components. Thus $\mathcal{H}_{2}$ has three elements

$$
\mathcal{H}_{2}=\left\{S_{i_{3}} \cup S_{i_{4}}, S_{i_{4}} \cup S_{i_{5}}, S_{i_{1}} \cup S_{i_{4}}\right\}
$$

corresponding to the three terms

$$
x_{2}^{2} x_{4} y_{3} y_{4}, \quad x_{2}^{2} x_{4} y_{1} y_{4} \quad \text { and } \quad x_{2}^{2} x_{4} y_{1} y_{4} .
$$

Acknowledgements The authors would like to thank Jim Propp and Lauren Williams for useful conversations related to this work. We thank the anonymous referees for their suggestions that have improved the exposition.

Note added in print In a sequel to the present paper [27], the authors give expansion formulas for the cluster variables in cluster algebras from arbitrary surfaces (allowing punctures) and prove the positivity conjecture for these cluster algebras.

\section{References}

1. Amiot, C.: Cluster categories for algebras of global dimension 2 and quivers with potential. Ann. Inst. Fourier (to appear)

2. Assem, I., Brüstle, T., Charbonneau-Jodoin, G., Plamondon, P.G.: Gentle algebras arising from surface triangulations. Preprint, arXiv:0903.3347

3. Buan, A., Marsh, R., Reineke, M., Reiten, I., Todorov, G.: Tilting theory and cluster combinatorics. Adv. Math. 204, 572-612 (2006). arXiv:math.RT/0402054

4. Buan, A.B., Marsh, R., Reiten, I.: Denominators of cluster variables. J. Lond. Math. Soc. 79(3), 589$611(2009)$ 
5. Caldero, P., Chapoton, F.: Cluster algebras as Hall algebras of quiver representations. Comment. Math. Helv. 81, 595-616 (2006). arXiv:math.RT/0410187

6. Caldero, P., Keller, B.: From triangulated categories to cluster algebras II. Ann. Sci. École Norm. Sup. (4) 39(6), 983-1009 (2006). arXiv:math.RT/0510251

7. Caldero, P., Keller, B.: From triangulated categories to cluster algebras. Invent. Math. 172, 169-211 (2008). arXiv:math.RT/0506018

8. Caldero, P., Zelevinsky, A.: Laurent expansions in cluster algebras via quiver representations. Mosc. Math. J. 6(3), 411-429 (2006). arXiv:math.RT/0604054

9. Caldero, P., Chapoton, F., Schiffler, R.: Quivers with relations arising from clusters ( $A_{n}$ case). Trans. Am. Math. Soc. 358(3), 1347-1364 (2006). arXiv:math.RT/0401316

10. Caldero, P., Chapoton, F., Schiffler, R.: Quivers with relations and cluster tilted algebras. Algebr. Represent. Theory 9(4), 359-376 (2006). arXiv:math.RT/0411238

11. Carroll, G., Price, G.: Unpublished result

12. Elkies, N., Kuperberg, G., Larsen, M., Propp, J.: Alternating-sign matrices and domino tilings (Part I). J. Algebr. Comb. 1(2), 11-132 (1992). arXiv:math/9201305

13. Fock, V., Goncharov, A.: Cluster ensembles, quantization and the dilogarithm. Preprint (2003). arXiv:math.AG/0311149

14. Fock, V., Goncharov, A.: Moduli spaces of local systems and higher Teichmüller theory. Publ. Math. Inst. Hautes Études Sci. 103, 1-211 (2006)

15. Fock, V., Goncharov, A.: Dual Teichmüller and lamination spaces. In: Handbook of Teichmüller Theory. Vol. I. IRMA Lect. Math. Theor. Phys., vol. 11, pp. 647-684. Eur. Math. Soc., Zürich (2007)

16. Fomin, S., Thurston, D.: Cluster algebras and triangulated surfaces. Part II: Lambda lengths. Preprint (2008). http://www.math.lsa.umich.edu/ fomin/Papers/cats2.ps

17. Fomin, S., Zelevinsky, A.: Cluster algebras I. Foundations. J. Am. Math. Soc. 15(2), 497-529 (2002) (electronic). arXiv:math.RT/0104151

18. Fomin, S., Zelevinsky, A.: Cluster algebras IV: Coefficients. Comput. Math. 143, 112-164 (2007). arXiv:math.RA/0602259

19. Fomin, S., Zelevinsky, A.: Unpublished result

20. Fomin, S., Shapiro, M., Thurston, D.: Cluster algebras and triangulated surfaces. Part I: Cluster complexes. Acta Math. 201, 83-146 (2008). arXiv:math.RA/060836

21. Fu, C., Keller, B.: On cluster algebras with coefficients and 2-Calabi-Yau categories. Trans. Am. Math. Soc. (to appear). arXiv:0710.3152

22. Gekhtman, M., Shapiro, M., Vainshtein, A.: Cluster algebras and Poisson geometry. Mosc. Math. J. 3(3), 899-934 (2003), 1199. arXiv:math.QA/0208033

23. Gekhtman, M., Shapiro, M., Vainshtein, A.: Cluster algebras and Weil-Petersson forms. Duke Math. J. 127(2), 291-311 (2005). arXiv:math.QA/0309138

24. Labardini-Fragoso, D.: Quivers with potentials associated to triangulated surfaces. Proc. Lond. Math. Soc. (to appear). arXiv:0803.1328

25. Musiker, G.: A graph theoretic expansion formula for cluster algebras of classical type. Ann. Comb. (to appear). arXiv:0710.3574

26. Musiker, G., Propp, J.: Combinatorial interpretations for rank-two cluster algebras of affine type. Electron. J. Comb. 14(1) (2007), Research Paper 15, 23 pp. (electronic). arXiv:math/0602408

27. Musiker, G., Schiffler, R., Williams, L.: Positivity for cluster algebras from surfaces. Preprint. arXiv:0906.0748

28. Palu, Y.: Cluster characters for triangulated 2-Calabi-Yau triangulated categories. Ann. Inst. Fourier 58(6), 2221-2248 (2008)

29. Propp, J.: Lattice structure for orientations of graphs. Preprint (1993). arXiv:0209.5005 [math]

30. Propp, J.: The combinatorics of frieze patterns and Markoff numbers. Preprint (2005). arXiv:math.CO/0511633

31. Schiffler, R.: A cluster expansion formula ( $A_{n}$ case), Electron. J. Comb. 15 (2008), \#R64 1. arXiv:math.RT/0611956

32. Schiffler, R.: On cluster algebras arising from unpunctured surfaces II. Adv. Math. (to appear). arXiv:0809.2593

33. Schiffler, R., Thomas, H.: On cluster algebras arising from unpunctured surfaces. Int. Math. Res. Not. 17, 3160-3189 (2009). arXiv:0712.4131

34. Sherman, P., Zelevinsky, A.: Positivity and canonical bases in rank 2 cluster algebras of finite and affine types. Mosc. Math. J. 4(4), 947-974 (2004), 982. arXiv:math.RT/0307082

35. Zelevinsky, A.: Semicanonical basis generators of the cluster algebra of type $A_{1}^{(1)}$. Electron. J. Comb. 14(1) (2007), Note 4, 5 pp. (electronic). arXiv:math.RA/0606775 